\title{
Can Thin Disks Produce Anomalous X-Ray Pulsars?
}

\author{
K. YAVUZ EKŞi $\dot{I}^{1,2}$ and M. ALI ALPAR ${ }^{2}$ \\ yavuz@sabanciuniv.edu, alpar@sabanciuniv.edu
}

\begin{abstract}
We investigate whether young neutron stars with fall-back disks can produce Anomalous X-Ray Pulsars (AXPs) within timescales indicated by the ages of associated supernova remnants. The system passes through a propeller stage before emerging as an AXP or a radio pulsar. The evolution of the disk is described by a diffusion equation which has self-similar solutions with either angular momentum or total mass of the disk conserved. We associate these two types of solutions with accretor and propeller regimes, respectively. Our numerical calculations of thin disk models with changing inner radius take into account the super-critical accretion at the early stages, and electron scattering and bound-free opacities with rich metal content. Our results show that, assuming a fraction of the mass inflow is accreted onto the neutron star, the fall-back disk scenario can produce AXPs for acceptable parameters.
\end{abstract}

Subject headings: accretion, accretion disks — pulsars: general — stars: neutron - X-rays: stars

\section{INTRODUCTION}

Anomalous X-Ray Pulsars (AXPs) (Mereghetti et al. 2002) are distinct from accretion powered X-ray pulsars in several aspects: (1) No binary companion or orbital Doppler modulations have been observed. (2) AXP rotation periods are clustered between 5-12 s while the periods of conventional X-ray pulsars span a much wider range $\left(P \sim 0.069-10^{4}\right.$ s). (3) They have large spin-down rates (Mereghetti \& Stella 1995) with a time-scale of $\tau=P / 2 \dot{P} \sim 10^{3}-10^{5}$ years, (4) Three of them have been associated with supernova remnants $(\mathrm{SNRs})$ indicating that they are young $\left(t_{S N R} \lesssim 5 \times 10^{4}\right.$ yrs.) (Gaensler et al. 2001;

\footnotetext{
${ }^{1}$ Boğaziçi University, 34342, Bebek, İstanbul, Turkey

${ }^{2}$ Sabancı University, Orhanl-Tuzla, İstanbul 34956, Turkey
} 
Tagieva \& Ankay 2003), (5) Luminosities of $L_{x} \sim 10^{35}-10^{36} \mathrm{ergs} \mathrm{s}^{-1}$ are well in excess of the spin-down power, (6) AXP spectra are soft compared to typical X-ray pulsars, with power law indices $\Gamma \gtrsim 2$. AXPs share all these features with Soft Gamma Ray Repeaters (SGRs) (Hurley 1999; Kouveliotou 1998, 1999). The observation of bursts from AXPs (Gavriil, Kaspi \& Woods 2002; Kaspi \& Gavriil 2002), suggests a strong connection between AXPs and SGRs.

In magnetar models (Duncan \& Thompson 1992; Thompson \& Duncan 1995, 1996) bursts are triggered and powered by enormous magnetic fields, $B \sim 10^{15}$ Gauss, and the energy source of the X-Ray emission is the decay of this magnetic field (Thompson \& Duncan 1995; Colpi, Geppert \& Page 2001). The star spins down by magnetic dipole radiation. While the magnetar model is quite successful in modelling the SGR and AXP bursts, and spindown rates, it can not explain the period clustering (Psaltis \& Miller 2002) except in one set of field decay models under special conditions (Colpi, Geppert \& Page 2001).

Accretion models for AXPs started with the work of van Paradijs, Taam \& van den Heuvel (1995). The current models invoke neutron stars with $B \sim 10^{12} \mathrm{G}$ and explain the period clustering in terms of asymptotic evolution of the neutron star rotation rate towards equilibrium with a fall-back disk (Chatterjee, Hernquist \& Narayan 2000, hereafter CHN; Alpar 2001; Marsden, Lingenfelter \& Rothschild 2001). These models require no binary companion. AXP ages are indicated by the ages of the supernova remnants associated with some AXPs. The possibility that some material in a supernova explosion might fall back and accrete onto the new-born neutron star has been explored by several authors (Colgate 1971; Scargle \& Pacini 1971; Roberts \& Sturrock 1973). Fall-back accretion disks have been invoked to address a diversity of astrophysical problems (Michel \& Dessler 1981, 1983; Michel 1988; Lin, Woosley \& Bodenheimer 1991). Mineshige et al. (1997) have shown, by using smoothed particle hydrodynamics (Monaghan 1992), that an accretion disk is formed around a new-born compact object if the progenitor had been rotating before the explosion. The total mass of the fall-back gas has been estimated to be $0.05 M_{\odot}$ by Hashimoto, Nomoto \& Shigeyama (1989), $0.1 M_{\odot}$ by Brown \& Bethe (1994) and $0.15 M_{\odot}$ by Chevalier (1989) (see also Lin, Woosley \& Bodenheimer (1991)).

The angular momentum carried by the neutron star and by the ambient material must play an important role in determining the subsequent evolution. Motivated by the recognition of angular momentum as an important initial parameter, Alpar (2001) proposed a classification of young neutron stars in terms of the absence or presence and properties of a fall-back disk. According to this model AXPs, SGRs and Dim Thermal Neutron Stars (DTNs) have similar periods because they are in an asymptotic spin-down phase in interaction with a fall-back disk. The different classes represent alternative pathways of neutron 
stars. Radio pulsars have no disks or encounter very low mass inflow rates while Radio Quiet Neutron Stars (RQNS) have such high mass inflow rates that their pulse periods are obscured by the dense medium around. AXPs and SGRs evolve through a propeller stage (Shvartsman 1970; Pringle \& Rees 1972; Illarionov \& Sunyaev 1975; Fabian 1975; Ikhsanov 2001) in which the rapid rotation of the neutron star prevents the inflowing matter from accreting onto the surface of the star and the star is spun-down until its rotation is slow enough that accretion can commence.

CHN pioneered a specific evolutionary model to produce AXPs with a fall-back disk. They employed the available time-dependent viscous thin disk models. There are upper limits and observations in the infrared and optical that have been evaluated within the CHN thin disk model to constrain and argue against the fall-back disk models in general. Two points should be underlined here. First, the recent detections in the infrared (and optical) supports the existence of a disk (Israel et al. 2003). The magnetar models do not have a prediction for magnetospheric production of infrared photons, and the detections in IR are well in excess of the extrapolation of a neutron star surface black-body fit to the X-ray band. The second point is that if a disk is present, it is unlikely that it will be a thin disk in a stable state, as the sources are expected to spend significant parts of their evolution in a propeller phase in which mass that can not be accreted to the neutron star will remain bound around the star and disk. In addition, there will be irradiation effects on the disk. The thin disk models are probably not the realistic models to compare with the data. Nevertheless, as the only available working models, they warrant a careful study to decide whether interaction with a fall-back disk can lead to AXP periods and luminosities at the ages indicated by SNR-AXP associations. On the other hand optical pulsations with a large pulse fraction observed from $4 \mathrm{U} 0142+614$ (Kern \& Martin 2002) can not be explained by emission or reprocessing from a disk. This observation, in addition to its successful application to model SGR and AXP bursts, clearly favors magnetar models, while the period clustering has a natural explanation in disk models. It may be the case that the AXPs (which show bursts like SGRs) do have surface magnetic fields in the higher multipoles and a dipole field in the $10^{12} \mathrm{G}$ range interacting with a fall-back disk to provide the rotational equilibrium that leads to period clustering. Such a hybrid model in which AXPs and SGRs would be the rare objects that correspond to the high magnetic field and high angular momentum (fall-back disk) corner of the parameter space of initial conditions, makes the actual possibilities of disk models worth exploring.

An investigation of the CHN model was carried out by Francischelli \& Wijers (2002) who argued that with the bound-free opacities that are relevant, the evolution would not lead to AXPs. This work was carried out with a disk solution with inner boundary condition relevant for a disk losing mass by accretion whereas the system throughout its evolution is 
in the propeller regime.

We employ different thin-disk solutions for propeller and for accretor phases of the evolution, use electron scattering and bound-free (mostly the latter) opacities as appropriate and include a range of initial disk masses. In the next section we present the model equations, the special analytical solutions, and their relation to the numerical calculations for a finite disk. Results are presented in section 3, and discussed in the final section.

\section{THIN DISK MODELS}

The evolution of a viscous thin disk is governed by a non-linear diffusion equation (Pringle 1981) which accepts three self-similar solutions (Pringle 1974) extending down to $r=0$, if the disk opacity has a power-law dependence on density and temperature. One of these solutions is a simple power-law both in spatial and temporal variables and is irrelevant for fall-back disks which have freely expanding outer boundaries. The other two solutions can be associated with accretion and propeller regimes. In the solution which we associate with the accretion regime (Cannizzo, Lee \& Goodman 1990) the total angular momentum of the disk is constant $\left(\dot{J}_{d}=0\right)$ and mass of the disk decreases in time such that $\dot{M}_{d} \propto t^{-\alpha}$. This is the solution employed by CHN for all phases of AXP evolution. In the other solution which we associate with the propeller regime, the mass of the disk is constant $\left(\dot{M}_{d}=0\right)$ and angular momentum of the disk increases in time by the viscous torque at the inner radius such that $\dot{J}_{d} \propto t^{-\beta}$. Pringle (1981) mentioned that "such a solution might represent a disc around a magnetized star which is rotating sufficiently rapidly that its angular velocity exceeds the Keplerian velocity at the magnetosphere" - i.e. a propeller. A real disk starts at an inner radius $R_{m}$ determined by the magnetic moment $\mu$ and rotation rate $\Omega_{*}$ of the neutron star, and mass flow rate $\dot{M}(r)$. For both types of analytical solutions extending to $r=0$, the application to a real disk with finite inner radius $R_{m}$ requires a numerical treatment, which shows that the accretor solution (Cannizzo, Lee \& Goodman 1990) and the propeller solution (Pringle 1991) are good representations of the numerical solutions, the initial conditions being 'forgotten' after a brief transition period. One dimensional thin disk solutions necessarily suppress the two dimensional mass flow in the $r-z$ plane of a realistic disk model. In the case of accretor type analytical solutions, the mass flow $\dot{M}(r)$ is inward in the inner disk. In the propeller type analytical solutions $\dot{M}(r)$ is outward, with the mass loss rate of the disk $\dot{M}_{d} \equiv \dot{M}(r=0)=0$. (Pringle (1991) numerically shows that there are also propeller type solutions — with non-vanishing torque at the inner boundary - in which mass flow is inwards in the inner parts of the disk.) In the real propeller, constant disk mass would be sustained with mass inflow and outflow; and small fractions of the mass flow can 
accrete to the neutron star or escape the system. For opacities of the form $\kappa=\kappa_{0} \rho^{a} T^{b}$, thin disk equations (Frank, King \& Raine 1992) can be solved to yield the power law indices of the time dependence in the two analytical solutions:

$$
\dot{M}_{d} \propto t^{-\alpha}, \quad \alpha=\frac{18 a-4 b+38}{17 a-2 b+32}
$$

for the accretor type solution (Cannizzo, Lee \& Goodman 1990; Francischelli \& Wijers 2002); and

$$
\dot{J}_{d} \propto t^{-\beta}, \quad \beta=\frac{14 a+22}{15 a-2 b+28} .
$$

for the solution we associate with propeller regime (Pringle 1991). For electron scattering dominated disks $(a=b=0) \alpha=19 / 16$ and $\beta=11 / 14$, and for bound-free opacity dominated disks $(a=1, b=-7 / 2) \alpha=5 / 4$ and $\beta=18 / 25$. CHN used $\alpha=7 / 6$ for analytical convenience.

The total torque is the sum of accretion torque $\dot{J}_{a}=r^{2} \Omega \dot{M}$ and the viscous torque $\dot{J}_{\nu}=2 \pi r^{2} W$ where $W$ is the vertically averaged viscous stress. The accretor solution is not precisely the time-dependent version of the Shakura \& Sunyaev (1973) solutions with the same inner boundary condition. The latter solutions assume vanishing viscous torque whereas the time-dependent solution implies vanishing total torque at the inner boundary.

The inner boundary of the disk, $R_{m}$, is where the ram pressure $P_{\text {ram }}=\rho v^{2}$ of the disk is balanced by the magnetic pressure $P_{m a g}=B^{2} / 8 \pi$. Assuming a dipole field for the neutron star, the magnetic pressure can be written as (Lipunov 1992):

$$
P_{m a g}= \begin{cases}\frac{\mu^{2}}{8 \pi r^{6}} & \text { if } r \leq R_{L} \\ \frac{\mu^{2}}{8 \pi R_{L}^{4} r^{2}} & \text { if } r>R_{L}\end{cases}
$$

where $R_{L}=c / \Omega_{*}$ is the light cylinder radius and $\mu$ is the dipole magnetic moment. We write the ram pressure as

$$
P_{\text {ram }}= \begin{cases}\frac{\dot{M}}{4 \pi r^{2}} \sqrt{2} \Omega_{K} r & \text { if } r \leq R_{c o} \\ \frac{\dot{M}}{4 \pi r^{2}} \sqrt{2} \Omega_{*} r & \text { if } r>R_{c o} .\end{cases}
$$

(see Romanova et al. (2003)) where $R_{c o}=\left(G M / \Omega_{*}^{2}\right)^{1 / 3}$ is the corotation radius and $\dot{M}$ is the mass flow rate. Here $\Omega_{K}$ denotes the Keplerian rotation rate at the inner edge of the disk. The two expressions we adopt for $P_{\text {ram }}$ are asymptotic forms for $\omega_{*} \ll 1$ and $\omega_{*} \gg 1$ where $\omega_{*}=\Omega_{*} / \Omega_{K}\left(R_{m}\right)$ is the fastness parameter.

When the flow rate at $R_{m}$ is greater than the critical rate $\dot{M}_{E d d}=4 \pi m_{p} c R_{m} / \sigma_{T}$, we follow Shakura \& Sunyaev (1973) and assume that the disk inside the "spherization" radius 
$R_{s}=\sigma_{T} \dot{M} / 4 \pi m_{p} c$ regulates itself as

$$
\dot{M}(r)=\frac{r}{R_{s}} \dot{M}_{E d d} \quad \text { if } r \leq R_{s}
$$

with the result that the total luminosity of the disk does not exceed the Eddington limit considerably (Lipunov 1992; Lipunova 1999). These considerations are relevant for the earliest stages of the mass flow typically extending over $\sim 20$ years in our calculations and do not effect the later period evolution of the star significantly. However, without such an incorporation of radiation pressure, the initial inner radius of the disk is obtained to be smaller than the radius of the neutron star, which is unphysical, and the system passes through an accretor stage. With the implementation of radiation pressure effects, the initial inner radius of the disk is greater than the corotation radius and the system is in a supercritical propeller stage. In the numerical calculations, the inner radius $R_{m}=R_{m}\left(\dot{M}, \Omega_{*}\right)$ is determined self-consistently at each time step using equations (3), (4) and (5).

The accretor solution with the boundary condition $\dot{J}_{d}=0$ had been applied for fallback disks by Mineshige, Nomoto \& Shigeyama (1993); Menou, Perna \& Hernquist (2001); Perna, Hernquist \& Narayan (2000), and CHN. Starting from the early phases, throughout most or all of its evolution into the asymptotic "tracking" AXP phase, the disk is in the propeller regime. We employ the relevant propeller type disk solution. This follows the power-law solution for $\dot{J}_{d}$ given in Eq. (2). In these propeller solutions at the inner boundary $\dot{M}\left(R_{m}\right)=0$ while in the accretion regime $\dot{M}\left(R_{m}\right)=\dot{M}_{d}$. We shall define a representative value $\dot{M}$ of the mass flow rate in the inner disk from the propeller torque $\dot{J}_{d}$ translating the torque $\dot{J}_{d} \propto t^{-\beta}$ to a flow rate such that

$$
\dot{J}_{d} \propto \sqrt{G M R_{m}} \dot{M}
$$

This yields a representative mass flow rate $\dot{M} \propto t^{-\alpha_{P}}$, defining the effective index $\alpha_{P}$. We emphasize that this definition is an estimate of the mass flow rates within the disk while the disk mass remains constant, with no mass loss from boundary. By employing the solution with a constant disk mass, we implicitly assume that the inflowing matter flung away by the magnetosphere of the neutron star returns back to the disk somewhere away from the inner boundary of the disk.

The critical fastness parameter for transition from propeller to accretor regime is assumed to be $\omega_{c r} \gtrsim 1$ and we take it to be 1 in all numerical experiments. The fastness parameter at which the torque vanishes is the equilibrium fastness parameter $\omega_{e q}$ and is inferred to be somewhat smaller than $\omega_{c r}$ as there are systems, like AXPs in our model, which are spinning down while accreting. We have observed numerically that the results do not depend sensitively on the value of $\omega_{e q}$ and we take it to be 0.7 . The range $\omega_{e q}<\omega_{*}<\omega_{c r}$ 
is not well interpreted with either the propeller or the accretor type of disk solutions, so when the fastness parameter $\omega_{*}$ has decayed to $\omega_{c r}$, we try two alternatives: (i) switching to the accretor solution (arguing $\dot{M} \neq 0$ ), (ii) continuing with the propeller solution (arguing $\dot{J} \neq 0$ ). In both cases we assume that a small fraction $\eta=\dot{M}_{\text {accreted }} / \dot{M}$ of the mass flow at the magnetic radius can reach the surface of the neutron star in order that $L=G M \eta \dot{M} / R_{*}$ gives the AXP luminosities.

Francischelli \& Wijers (2002) showed that the disk is electron scattering dominated only in the initial stages when $\dot{M}_{17}(t)>50[(1+X) / Z]^{-7 / 10} B_{12}^{3 / 5}$, while later on, at lower mass flow rates, bound-free opacity dominates. Here $\dot{M}_{17}=\dot{M} / 10^{17} \mathrm{~g} \mathrm{~s}^{-1}, B_{12}=B / 10^{12}$ Gauss, $X$ is the hydrogen mass fraction and $Z$ is the heavy element mass fraction. Assuming the disk to be rich in heavy elements, we choose $X=0.1$ and $Z=0.9$. The flow rate in the propeller solution declines with a softer power-law index than it does in the accretion solution because in the former case matter cannot accrete and the decrease in the flow rate is only by the viscous spreading of the disk.

We incorporate the torque model used by Alpar (2001) in the form

$$
\dot{J}_{*}= \begin{cases}\sqrt{G M R_{m}} \dot{M}\left(1-\omega_{*} / \omega_{e q}\right) & \text { for } \omega_{*}<\omega_{c r} \\ \dot{J}_{d}\left(1-\omega_{*} / \omega_{e q}\right) & \text { for } \omega_{*}>\omega_{c r} .\end{cases}
$$

As noted by Francischelli \& Wijers (2002) and Li (2002), this is a very efficient propeller torque model compared to some other available propeller torques obtained through simple energy or angular momentum arguments. This form of the torque is supported by the detailed numerical simulations of the magnetic boundary layers investigated by Daumerie (1996). Recently Ikhsanov (2001) revived the propeller model of Davies \& Pringle (1981) which incorporates a still more efficient torque $\dot{J} \propto-\omega_{*}^{2}$ for the sub-sonic propeller, and a torque $\dot{J} \propto-\omega_{*}$, which agrees with our model in the large $\omega_{*}$ limit, for the super-sonic propeller regime. These models do not entail asymptotic approach to rotational equilibrium, and are therefore not relevant for addressing the period clustering of AXPs. The recent numerical work of Romanova et al. (2003) also indicate an efficient propeller torque $\left(\dot{J} \propto-\Omega_{*}^{4 / 3}\right)$. For $\omega_{*} \gg 1$ matter can be flung away with the tangential velocity of the field lines at $R_{m}$ which is greater than the escape velocity so that some mass loss will take place in disagreement with the propeller type solution we employ. This means that the remaining mass of the disk, when the system reaches $\omega_{*}=\omega_{c r}$ and accretion onto the neutron star is allowed, is actually lower than what we find by assuming the mass of the disk is conserved. In this case the factor $\eta=\dot{M}_{\text {accreted }} / \dot{M}$ need not be very low but can attain a reasonable value.

The initial mass of the disk can be found by integrating the mass flow rate after accretion starts. This yields $M_{d}=t_{t r} \dot{M}_{t r} /(\alpha-1)$ where $t_{t r}$ is the transition time from propeller to 
the accretor regime and $\dot{M}_{t r}$ is the inflow rate at $t=t_{t r}$, and $\alpha=5 / 4$. By fitting to the numerical solutions we have determined, for an initial mass flow rate of $5 \times 10^{27} \mathrm{~g} \mathrm{~s}^{-1}$, that $\dot{M}_{t r}=2 \times 10^{16} \times \mu_{30}^{1.96} \mathrm{~g} \mathrm{~s}^{-1}$ and $t_{t r}=10^{5} \times \mu_{30}^{-2}$ years which means that $M_{d} \cong 1.2 \times 10^{-4} M_{\odot}$. This nominal value of the initial disk mass is 50 times smaller than the nominal value incorporated in the $\mathrm{CHN}$ model. From our fits we find that the initial inflow rate and the initial mass of the disk are related through $M_{d} /\left(10^{-4} M_{\odot}\right)=0.2074 \dot{M}_{0} /\left(10^{27} \mathrm{~g} \mathrm{~s}^{-1}\right)+0.1812$ for our parameter range.

\section{RESULTS}

We have followed the evolution of the star-disk system for $10^{5}$ years, solving the torque equation (7) numerically with the adaptive step-size Runge-Kutta method (Press et al. 1992). We assumed the radius and mass of the neutron star to be $R_{*}=10^{6} \mathrm{~cm}$ and $M_{*}=1.4 M_{\odot}$, respectively. In all numerical experiments we have fixed the initial period $P_{0}=15 \mathrm{~ms}$, and considered dipole magnetic moments in the range $\mu_{30}=1-10$. Electron scattering opacity prevails in the disk at the high initial flow rates. The system is initially in the super-critical propeller stage (i.e. radiation pressure is dominant). To follow one example, for an initial mass flow rate $5 \times 10^{27} \mathrm{~g} \mathrm{~s}^{-1}$ which corresponds to an initial disk mass of $1.2 \sim 10^{-4} M_{\odot}$ and the inner radius is at $R_{m}=31 R_{*} \mu_{30}^{1 / 3}\left(P_{0} / 15 m s\right)^{1 / 6}$. The dynamical time-scale $T_{d} \sim \Omega_{K}^{-1}$ at this radius is $1.23 \times 10^{-2} \mu_{30}^{1 / 6}\left(P_{0} / 15 \mathrm{~ms}\right)^{1 / 12} \mathrm{~s}$. For $\mu_{30}=5$ the supercritical propeller stage lasts for $\simeq 15$ years. In this stage the effective power law index, $\alpha_{p}$ is equal to $\beta=11 / 14$, because the disk inner radius does not depend on $\dot{M}_{0}$ in the super-critical regime, so that $\dot{M}=10^{27}\left(t / T_{d}\right)^{-11 / 14}$ (see Eq. (6)). After $\simeq 15$ years radiation pressure ceases to be important. Electron scattering continues as the dominant opacity until $t \simeq 75$ years; in this stage the effective power law index is 55/63. After $t \simeq 75$ years, the propeller regime continues with bound-free opacity dominating in the disk; the effective power law index is now $\alpha_{p}=4 / 5$ and remains so as long as the propeller regime continues. At around $10^{4}$ years the fastness parameter drops below $\omega_{c r}$ and the system enters the "tracking phase" CHN. It is during this period that we observe the system as an AXP.

In Figure 1 we give the period evolution of a neutron star for these parameters. These are the typical values leading to AXPs and SGRs. Panel (a) corresponds to the case in which we switched to the accretor type of solution when the fastness parameter decayed below $\omega_{c r}$ and panel (b) correspond to the case in which we continued with the propeller solution. We see that the latter case yields a somewhat more gradual period evolution because the equilibrium period increases less rapidly in this case. Figure 2 shows the evolutionary tracks in the $P-\dot{P}$ diagram. Again the two panels (a) and (b) correspond to the two cases as 
described for Figure 1. We see that the fall-back disk model can account for the position of AXPs and SGRs in the $P-\dot{P}$ diagram. In both cases the observed AXP luminosities require that the mass accretion rate onto the neutron star, $\dot{M}_{*}$, is only a fraction $\eta=\dot{M}_{*} / \dot{M}_{d} \sim 10^{-2}$ of the mass accretion rate in the disk. Whether the accreted fraction can be this low when the system is asymptotically close to equilibrium is an open question. The Soft X-Ray Transient Aql X-1 may have exhibited a comparably small accreted fraction of its mass transfer rate when it went through a reduction in luminosity by $10^{-2}-10^{-3}$, interpreted as a transition to the propeller state (Campana et al. 1998; Zhang, Yu \& Zhang 1998; Cui et al. 1998). Alternatively, the small value of $\eta$ implied by the present model calculations may reflect our assumption that the mass flow of the disk remains constant throughout the propeller phase: In reality some matter should have been expelled from the system which would lead to lower mass flow rates in the present day disk and hence the actual accreted fraction $\eta$, to meet the observed luminosities, could be higher. In any case models for an estimation of $\eta$ on the basis of accretion or propeller dynamics are not available at present (see Romanova et al. (2003) for a numerical approach). The result $\eta \sim 10^{-2}$ is an empirical constraint on fall-back disk models if they are to explain the observed luminosities of AXPs. Any independent evidence on the value of $\eta$ in these or similar sources will provide a test of the presently employed fall-back disk model.

Our results do not change qualitatively for initial disk masses $0.8-2.25 \times 10^{-4} M_{\odot}$. The transition to the accretion stage becomes earlier for greater initial disk masses. For $2.25 \times 10^{-4} M_{\odot}$ and $\mu_{30}=4$, the transition to the accretion regime occurs at 3000 years and for higher initial disk masses we obtain mass inflow rates as high as $10^{19} \mathrm{~g} \mathrm{~s}^{-1}$ which give $\eta \sim 10^{-3}$ to obtain present day luminosities of AXPs. For $M_{d}<0.8 \times 10^{-4} M_{\odot}$ the magnetic radius exceeds the light cylinder radius $R_{L}$ at an early stage and the source becomes a radio pulsar. The fact that the model gives radio pulsars at initial disk masses just below the range leading to AXPs and SGRs supports a continuity between AXPs, SGRs and radio pulsars, and suggests that radio pulsars might also have disks around them. We shall explore in a separate paper the possibilities of this continuity in initial conditions for different classes of neutron stars, in particular evolutionary tracks leading to AXPs, SGRs and radio pulsars in the same region of the $P-\dot{P}$ diagram as the AXPs (McLaughlin et al. 2003).

Thin disk models yield AXPs only if an efficient torque model is used as noted by Francischelli \& Wijers (2002) and Li (2002). Our simulations with other torque models (Fabian 1975; Ghosh \& Lamb 1978) show that these do not produce AXPs, verifying the results of Francischelli \& Wijers (2002) and Li (2002). However, as noted in the previous section, recent analytical (Ikhsanov 2001) and numerical (Daumerie 1996; Romanova et al. 2003) studies lend support to an efficient torque model. 
The question naturally arises as to why the disk evolution does not continue, to give sources with $P_{*}>12 \mathrm{~s}$; what is the justification for assuming that disk evolution stops at a finite age $\left(\sim 10^{5}\right.$ yrs in the present work $)$ ? The formal power-law solutions predict disk masses/torques decaying to infinitely small values as $t \rightarrow \infty$. In reality at low density and temperature the disk undergoes a transition to a neutral state, stopping its viscous evolution. Instabilities are likely to clump and destroy the disk eventually. As estimated by Menou, Perna \& Hernquist (2001) fall-back disks become neutralized at around $10^{4}$ years. In our models, the slower decrease in disk density and temperature leads to neutrality at around $10^{5}$ years. CHN gave an explanation for the upper cutoff of the spin period in terms of the transition from a thin disk to an advection dominated accretion flow with mass loss.

\section{DISCUSSION}

Our results show that for thin disks with bound-free opacities prevailing most of the time and with a range of initial disk masses $\left(M_{d} \sim 10^{-4} M_{\odot}\right)$ and neutron star dipole magnetic moments $\left(\mu_{30}=1-10\right)$ neutron stars do end up in the period range $P=5-15 \mathrm{~s}$ at ages $10^{4}-10^{5}$ years. The model we introduced yields present day disks that are consistent with the infrared observations for AXPs and SGRs (see, eg. Israel et al. (2003); Eikenberry et al. (2001) and references therein), as we shall detail in a subsequent paper. The observed luminosities require that only a fraction of the mass flow rate is accreted onto the neutron star in the current asymptotic phase.

The radial (Sunyaev \& Shakura 1977) and temporal (Pringle 1991) properties of propeller disks are different from the properties of disks in the mass-loss "accretion" regime. The flow rate in the accretor solutions for the disk decreases steeply both by losing mass due to accretion and by the viscous spreading of the disk, whereas in the propeller solutions it is only the "thinning" of the disk by viscous spread that decreases the flow rate, while the disk mass remains constant. It is possible that a fall-back disk loses mass also in the propeller regime because of the propeller effect itself, depending on the fastness parameter. The simple power-law models for the flow rate are working models which do not take into account the coupling of the star to the structure of the disk. As shown by Francischelli \& Wijers (2002) whether one obtains an AXP or not depends sensitively on the power-law index $\alpha$. Extending the analysis to disk evolution in the propeller regime provides favorable effective power-law indices $\alpha_{p}<1$.

We conclude that fall-back disks may well lead to the present day properties of AXPs and SGRs, even in the available thin disk models within the options of simple power law solutions. A more realistic treatment would include effects of the magnetic field on disk 
evolution, realistic opacities, including the iron line opacity (Fryer, Colgate \& Pinto 1999) for the fall-back disk which is rich in heavy elements, and the dependence of the accretion rate itself on the conditions at the inner boundary of the disk, including the effects of the motion of the inner radius (Spruit \& Taam 1993). All of these effects would result in time evolution of the disk that cannot be described by simple power laws. Taking account of the complexities of the real problem is not likely to produce qualitative disagreement with the evolutionary timescales and tracks in the $P-\dot{P}$ diagram, as long as the torque is efficient.

The fall-back disk models do not provide an explanation for the AXP and SGR bursts or for the optical pulsations observed in 4U 0142+614 (Kern \& Martin 2002). These phenomena are likely to originate with processes in the crust or magnetosphere of a neutron star with a strong magnetic field. We have shown that a fall-back disk around a neutron star provides a viable mechanism to get the observed period range at the AXP ages, provided that a fraction $\eta \sim 10^{-2}$ of the mass flow is accreted. Applying this model in conjunction with magnetar models for the bursts and optical pulsations requires that the magnetar fields on the surface of the neutron star are in higher multipoles. As we have shown, the observed spindown rates and history of the AXPs (or SGRs) are determined by disk torques rather than magnetic dipole models. Such a hybrid model assumes that magnetospheric generation of optical pulsations around a neutron star with a surface dipole field of $\sim 10^{12} \mathrm{G}$ and higher multipoles of $\sim 10^{14}-10^{15} \mathrm{G}$ is possible in the presence of the fall-back disk. A demonstration of such a magnetospheric model is beyond the scope of the present paper, and is to be attempted in future work. We finally note that the extended quiet (low-noise) spindown episodes of AXPs is compatible with the behavior of accreting sources, in that the same AXPs have had high-level noise characteristic of accreting sources; and there are well known accreting sources like 4U 1907+09 which exhibit similar extended quiet spindown (Baykal et al. 2001).

We thank M.Hakan Erkut, Ünal Ertan, Ersin Göğüs and R.A.M.J. Wijers for useful discussions, the referee for helpful comments and E.N. Ercan for her support and encouragement. This work was supported by Boğaziçi University Research Foundation under code 01B304 for KYE, by the Sabancı University Astrophysics and Space Forum, by the High Energy Astrophysics Working Group of TÜBİTAK (The Scientific and Technical Research Council of Turkey) and by the Turkish Academy of Sciences for MAA.

\section{REFERENCES}

Alpar, M.A., 2001, ApJ, 554, 1245. 
Baykal, A. et al., 2001, MNRAS, 327, 1269.

Brown, G.E. \& Bethe, H.A., 1994, ApJ, 423, 659.

Campana, S. et al. 1998, ApJ, 499, L65.

Cannizzo, J.K., Lee, H.M. \& Goodman, J., 1990, ApJ, 351, 38.

Chatterjee, P., Hernquist, L. \& Narayan, R., 2000, ApJ, 534, 373.

Chevalier, R.A., 1989, ApJ, 346, 847.

Colgate, S.A., 1971, ApJ, 163, 221.

Colpi, M., Geppert, U. \& Page, D., 2000, ApJ, 529, L29.

Cui, W., Barret, D., Zhang, S.N., Boirin, L. \& Swank, J., 1998, ApJ, 502, L49.

Daumerie, P.R., 1996, PhD thesis, University of Illinois, Urbana-Champaign.

Davies, R.E., Pringle, J.E., 1981, MNRAS, 196, 209.

Duncan R.C. \& Thompson, C., 1992, ApJ, 392, L9.

Eikenberry, S.S. et al. 2001, ApJ, 563, L133.

Fabian, A.C., 1975, MNRAS, 173, 161.

Francischelli, G.J. \& Wijers, R.A.M.J., ApJ, submitted, (Astro-ph/0205212).

Frank, J., King, A. \& Raine, D., 1992, Accretion Power in Astrophysics, Cambridge.

Fryer, C.L., Colgate, S.A. \& Pinto, P.A., 1999, ApJ, 511, 885.

Gavriil, F.P., Kaspi, V.M. \& Woods, P.M., 2002, Nature, 419, 142.

Gaensler, B.M., Slane, P.O., Gotthelf, E.V. \& Vasisht, 2001, ApJ, 559, 963.

Ghosh, P., \& Lamb, F.K., 1978, ApJ, 223, L83

Hashimoto, M, Nomoto, K. \& Shigeyama T., 1989, A\&A, 210, L5.

Hurley, K., 1999, AIP Conf. Proc. 510, Fifth Compton Symp., ed. M.L. McConnell \& J. M. Ryan (New York: AIP), 515.

Ikhsanov, N.R., 2001, A\&A, 368, L5. 
Illarionov, A.F. \& Sunyaev, R.A., 1975, A\&A, 39, 185.

Israel, G.L. et al., 2003, ApJ, 589, L93.

Kaspi, V.M. \& Gavriil, F.P., 2002, IAU Circ., 7924.

Kern, B. \& Martin, C., 2002, Nature, 417, 571.

Kouveliotou, C. et al. 1998, Nature, 393, 235.

-. 1999,ApJ, 510, L115.

Li, X.-D., 2002, ApJ, 579, L37.

Lin, D.N.C., Woosley, S.E. \& Bodenheimer, P.H., 1991, Nature, 353, 827.

Lipunov, Astrophysics of Neutron Stars, Berlin: Springer.

Lipunova, G.V., 1999, Astron.Lett., 25, 508.

Marsden, D, Lingenfelter, R.E., Rothschild, R.E., \& Higdon, J.C., 2001, ApJ, 550, 397.

McLaughlin, M.A. et al., 2003, ApJletters accepted, astro-ph/0306065.

Menou, K., Perna, R. \& Hernquist, L., 2001, ApJ, 559, 1032.

Mereghetti, S., Chiarlone, L., Israel, G.L., \& Stella, L., 2002, in Neutron Stars, Pulsars, and Supernova Remnants, ed. W. Becker, H. Lesch, \& J. Trümper (MPE Rep. 278; Garching: MPE), 29

Mereghetti, S. \& Stella, L, 1995, ApJ, 442, L17.

Michel, F.C., 1988, Nature, 333, 644.

Michel, F.C. \& Dessler, A.J., 1981, ApJ, 251, 654.

Michel, F.C. \& Dessler, A.J., 1983, Nature, 303, 48.

Mineshige, S., Nomoto, K., \& Shigeyama, T., 1993 A\&A, 267, 95.

Mineshige, S., Nomura, H., Hirose, M., Nomoto, K. \& Suzuki, T., 1997, ApJ, 489, 227.

Monaghan, J.J., 1992, ARA\&A, 30, 543.

Perna R., Hernquist, L. \& Narayan, R., 2000, ApJ, 541, 344. 
Press, WH., Teukolski, S.A., Vetterling, W.T. \& Flannery, B.P., 1992, Numerical Recipes in $C$, Cambridge.

Pringle, J.E., 1974, Ph.D. thesis, Univ. Cambridge.

Pringle, J.E., 1981, ARA\&A, 19, 137.

Pringle, J.E. 1991, MNRAS, 248, 754.

Pringle, J.E. \& Rees, M.J., 1972, A\&A, 21, 1.

Psaltis, D. \& Miller, M.C., 2002, ApJ578, 325.

Romanova, M.M., Toropina, O.D., Toropin, Yu.M. \& Lovelace, R.V.E., 2003, ApJ, 588, 400.

Roberts, D.H. \& Sturrock, P.A., 1973, ApJ, 251, 654.

Scargle, J.D. \& Pacini, F., 1971, Nature Phys. Sci., 231, 144.

Shakura, N.I. \& Sunyaev, R.A., 1973, A\&A, 24, 337.

Shvartsman, V.F., 1970, Radiofizika, 13, 1852.

Spruit, H., \& Taam, R.E., 1993, ApJ, 402, 593.

Sunyaev \& Shakura 1977, Pis'ma Astron. Zh., 3, 262.

Tagieva, S. \& Ankay, A., 2003, Astron.Astrophys. Trans., 20.

Thompson, C. \& Duncan, R.C., 1995, MNRAS, 275, 255.

Thompson, C. \& Duncan, R.C., 1996, ApJ, 473, 322.

van Paradijs, J., Taam, R.E., \& van den Heuvel, E.P.J. 1995, A\&A, 299, L41.

Zhang, S.N., Yu, W., Zhang, W., 1998, ApJ, 494, L71. 

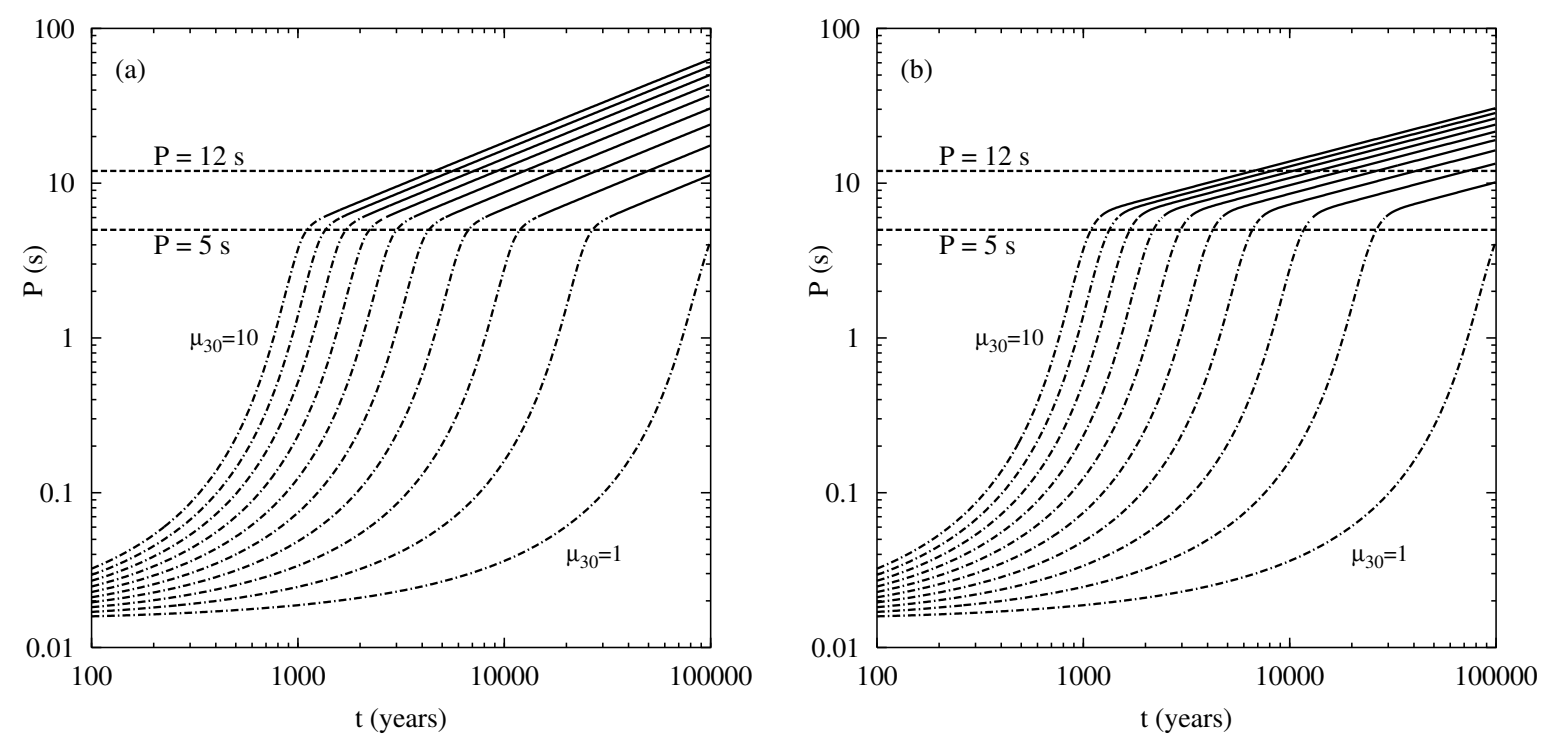

Fig. 1. - Period evolution of a neutron star with mass $M=1.4 M_{\odot}$, initial period $P_{0}=0.015$ s and initial disk mass $M_{0}=1.2 \times 10^{-4} M_{\odot}$. The values of magnetic dipole moments $\mu_{30}$ are $1,2, \ldots, 10$, with $\mu_{30}=1$ for the lowermost curve and $\mu_{30}=10$ for the uppermost. The dashed and dotted lines show the rapid spindown phase while the solid line stand for the "tracking" stage. The dashed horizontal lines show the period range of AXPs and SGRs. Neutron stars with higher magnetic dipole moments reach the tracking phase earlier. The panels correspond to the two options we used for $\omega_{*}<\omega_{c r}$. In both cases $\omega_{c r}=1$ and $\omega_{e q}=0.7$. In case (a) the disk evolves with the accretor solution for $\omega_{*}<\omega_{c r}$, and in case (b) it continues to evolve with the propeller solution. 

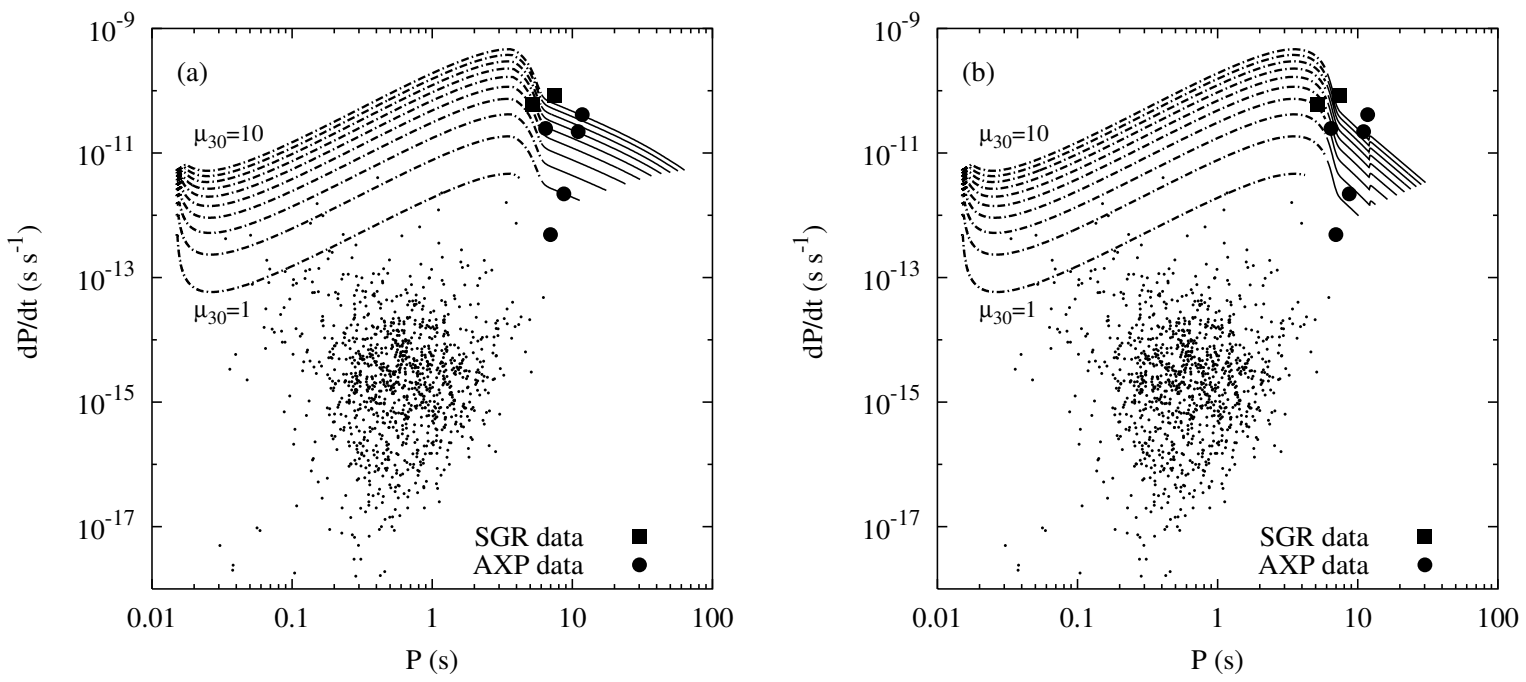

Fig. 2.- Evolutionary tracks in the $P-\dot{P}$ diagram with the same parameters as in Figure1. The two panels again correspond to the two cases in which (a) the disk switches to the accretor solution, and (b) it proceeds with the propeller solution. The dashed and dotted lines show the rapid spindown phase while the solid line stand for the "tracking" stage. The dots denote the radio pulsars. AXPs are shown by filled circles and SGRs by squares. With different $\mu_{30}$ and/or initial mass values of the disk, the model can cover all AXPs and SGRs. 